\title{
Neuere Erwerbungen der Coleopterenabteilung des Königlichen zoologischen Museums in Berlin.
}

\section{Die Fiorische Coleopterensammlung.}

Das Königliche Museum in Berlin erwarb kürzlich die Fior is che Coleopterensammlung. Diese Sammlung repräsentiert die Coleopterenfauna Italiens fast lückenlos. Und nicht nur die Arten, sundern auch die als Subspezies ausgebildeten geographischen Formen vieler Arten sind vorhanden. Wir finden hier z. B. von Carabus coriaceus die Subspezies cordicollis Motsch., basilicatus Born, mediterraneus Born, die man sonst in Museen und anderen Sammlungen nicht oder nur vereinzelt $\mathrm{zu}$ sehen gewohnt ist. Dasselbe gilt von Carabus violaceus romanus Born und Fiorii Born, C. Rossii Pirazzolii Géh. und Costae Géh., C. Lefeburei Leonii Born, C. clathratus arelatensis Lopouge, C. italicus Rostagnoi Luig. und Ronchettii Born, C.hortensis calabrus Fiori, C. concolor tendanus Born, omensis Born, bernensis Born, heteromorphus Dan., sturensis Born, Steckii Born usw. Auch die Cicindela Fiorii befindet sich in der Sammlung.

Die Fiorische Sammlung ist reich an typischen Stücken zahlreicher Arten aus älterer und neuerer Zeit, besonders an Arten und Unterarten, welche von Bernhauer, Born, Daniel, Ganglbauer, Holdhaus, Reitter, O. Schneider, Lopez, Baudi, Luigi, Porta, Dodero, Alzona, Leoni, Fracassi, Ragusa und von Fiori selbst beschrieben sind. Das reiche originale Material, welches Fiori, Professor in Bologna, zu seinen Abhandlungen über die Coleopteren Italiens benutzt hat, befindet sich natürlich in dieser Sammlung. Seine Abhandlungen über die Coleopteren Italiens hat F i or i veröffentlicht in den „Atti della Società dei Naturalisti di Modena“, im "Bulletino della Società entomologica italiana" (Firenze), in der "Revista coleotterologica italiana" (Camerino) und im "Naturalista Siciliano" durch viele Jahrgänge hindurch.

Wie artenreich die Gattungen in der Sammlung vertreten sind, geht aus der Vorführung einiger Stichproben hervor. Von den italienischen Arten der in 26 Arten vertretenen Gattung Anophthalmus sind 20 vorhanden, von Pterostichus (mit Poecilus) 88 Arten ohne die vielen Subspezies, von Abax (inkl. Percus) 22, von Amara 51, Ophonus 27, Harpalus 45, Bembidium 90 Arten. Einen Schatz an Arten bietet die Familie der Pselaphiden (145 Arten), darunter Typen aus den Gattungen Trimium, Dimarus, Euplectus, Trogaster, Amaurops, Reichenbachia, Bythinus und Pselaphus. Ganz vorzüglich sind die Staphyliniden vertreten (Atheta allein 
mit 115 Arten). Unter den artenreichen Malacodermaten sind besonders die Gattungen Malthinus und Malthodes bezüglich ihrer grofsen Artenzahl hervorzuheben. Alle die vielen Familien zeigen uns, dafs die Gattungen und Arten von Sachkundigen durchgearbeitet sind. Die Arten sind sämtlich benannt und die meisten in mehreren Exemplaren vertreten, wobei die Mannigfaltigkeit der Fundorte und die Variabilität der Individuen als Leitmotive gedient haben.

Ein nicht zu unterschätzender Wert steckt in dem Vorhandensein der in italienischen Exemplaren vertretenen zahlreichen mitteleuropäischen Arten, da sonst in mitteleuropäischen Sammlungen die südeuropäischen Angehörigen mitteleuropäischer Arten wenig vertreten sind. Darin liegt der tiergeographische Wert dieser italienischen Sammlung für das Berliner Königliche Museum.

Dazu kommen die zahlreichen Arten, Unterarten und Varietäten der italienischen Fauna, welche dem Königlichen Museum bisher noch fehlten.

Der Umfang der Sammlung ist daraus zu entnehmen, dafs sie in 145 Kästen untergebracht ist, deren Grölsendimensionen $34 \mathrm{zu} 45 \mathrm{~cm}$ betragen. In den Kästen der kleinen und kleinsten Coleopteren befinden sich je 500-600 und mehr Exemplare.

Was den Zustand der Objekte betrifft, so kann man nicht nur die ausgezeichnete Konservierung derselben, sondern auch die äufserst subtile Präparation jedes Individuums bewundern. Besonders ist dies für die sehr kleinen auf kleinen Kartons angebrachten Objekte hervorzuheben. Auch die Etikettierung sämtlicher Exemplare ist von Anfang bis zu Ende tadellos.

Die Erwerbung der Fiorischen Sammlung für das Königliche zoologische Museum in Berlin ist daher als eine sehr wertvolle zu bezeichnen. Herrn E. von Bodemeyer verdanken wir die Vermittelung derselben.

H. K olb e.

H. Bickhardt, der Herausgeber der Coleopterolog. Blätter, wohnt ab 1. Juli 1911:

Kassel, Elfbuchenstrafse 32.

Als neues Mitglied wurde durch die Herren Apotheker P. K uhnt und G. Reine $\mathrm{ck}$ vorgeschlagen :

Herr Max Bräutigam, Berlin N. 65, Triftstrafse 49. 


\section{$2 \mathrm{BHL}$ Biodiversity Heritage Library}

Kolbe, H. J. 1911. "Neuere Erwerbungen der Coleopterenabteilung des Königlichen zoologischen Museums in Berlin." Deutsche entomologische Zeitschrift 1911(4), 495-496. https://doi.org/10.1002/mmnd.48019110423.

View This Item Online: https://www.biodiversitylibrary.org/item/103379

DOI: https://doi.org/10.1002/mmnd.48019110423

Permalink: https://www.biodiversitylibrary.org/partpdf/236081

\section{Holding Institution}

Harvard University, Museum of Comparative Zoology, Ernst Mayr Library

\section{Sponsored by}

Biodiversity Heritage Library

\section{Copyright \& Reuse}

Copyright Status: Public domain. The BHL considers that this work is no longer under copyright protection.

This document was created from content at the Biodiversity Heritage Library, the world's largest open access digital library for biodiversity literature and archives. Visit BHL at https://www.biodiversitylibrary.org. 